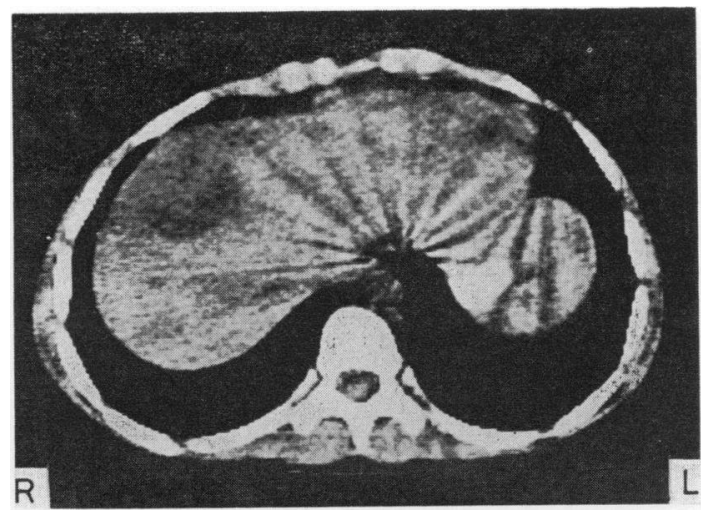

Figure Computed tomogram showing a solitary lesion in the right lobe of liver.

density lesions of various sizes in the right lobe, with one larger lesion (Figure).

At laparotomy two abscesses deep in the right lobe were found, and each drained $20 \mathrm{cc}$ of pus. Staphylococcus aureus was isolated in the pus and appropriate antibiotics were given. Apart from developing a sterile right pleural effusion, her recovery was smooth.

\section{Discussion}

Liver abscess in children remains a difficult diagnostic problem owing to both its rarity and its non-specific clinical features. As late as 1977 reports still indicated that the diagnosis in over $60 \%$ of cases was not made until necropsy, despite the presence of fever and hepatomegaly. ${ }^{1}$ In our experience only prolonged unexplained fever is a consistent symptom, and even abdominal pain may be absent or minimal. Right upper quadrant tenderness is another clinical clue. Blood tests indicating leucocytosis and altered liver function are helpful when positive but are more commonly negative even if there is extensive hepatic sepsis. Isotope liver scan has been most commonly used for diagnosis but is being increasingly criticised for its low sensitivity. Ultrasound assessment represents an improvement but its accuracy depends on interpretative skills, as indicated by our second patient whose first scan was reported as normal. Computed tomography promises to be more sensitive ${ }^{5}$ and our third patient may represent the first documented application in diagnosis of liver abscess in children.

The management of nonamoebic liver abscess is surgical and the approach should be individual. As in our first patient, cholangitis may be an underlying cause, necessitating common duct exploration for satisfactory eradication of the disease. Chronic reactive changes after prolonged antibiotics may render the lesions unresponsive to simple drainage, requiring resection as in our second child. Finally, there is no substitute for laparotomy and careful palpation for detection of liver abscess, as even ultrasound and computed tomography may not be able to show all important lesions.

\section{References}

1 Cushid MJ. Pyogenic hepatic abscess in infancy and childhood. Pediatrics 1978;62:554-9.

2 Larsen LR, Raffensperger J. Liver abscess. J Pediatr Surg 1979;14:329-31.

3 Harrington E, Bleicher MA. Cryptogenic hepatic abscess in two uncompromised children. J Pediatr Surg 1980;15: 660-2.

4 Arya LS, Ghani R, Abdali S, Singh M. Pyogenic liver abscesses in children. Clin Pediatr (Phila) 1982;21:89-93.

5 Buchman TG, Zuidema GD. The role of computerized tomographic scanning in the surgical management of pyogenic hepatic abscess. Surg Gynecol Obstet 1981 ;153: $1-9$.

Correspondence to Dr P K H Tam, Division of Paediatric Surgery, Department of Surgery, University of Hong Kong, Queen Mary Hospital, Hong Kong.

Received 15 June 1983

\title{
Selective placement of bronchial suction catheters in intubated neonates
}

\author{
M PLACZEK AND M SILVERMAN
}

Department of Paediatrics and Neonatal Medicine, Royal Postgraduate Medical School, Hammersmith Hospital, London

SUMMARY Flexible suction catheters were passed through the endotracheal tubes of infants undergoing mechanical ventilation, just before chest radiographic examination for clinical purposes. With the head straight, 7 of 10 straight catheters entered the right main bronchus but with the head turned, 17 of 20 straight catheters and 19 of 20 curved tip catheters entered the contralateral bronchus.
Mechanical ventilation of newborn babies is not infrequently accompanied by pulmonary atelectasis related to mechanical ventilation, 12 or tracheal suction, ${ }^{3}$ or after extubation. ${ }^{2}$ It occurs more commonly in low birthweight infants ${ }^{1}$ and after prolonged periods of intubation. ${ }^{2}$ The management of lobar atelectasis requires prompt recognition of the problem, suctioning of the tracheobronchial tree, and reinflation of the affected lung. 
When a catheter is advanced beyond the trachea the direction it takes depends mainly on the angle that the bronchi make with the trachea. In older children, with the head in the midline, a straight catheter more frequently enters the right main bronchus, although the use of an angulated catheter ${ }^{4}$ or head turning to the right ${ }^{5}$ will facilitate entry into the left main bronchus. We evaluated the effects of head position and catheter type (straight or curved tip) for selective bronchial intubation with suction catheters in intubated newborn infants.

\section{Patients and methods}

Forty two newborn infants (gestational age 26-40 weeks; birthweight $800-3880 \mathrm{~g}$ ) requiring mechanical ventilation for a variety of conditions were studiedsome on several occasions during the course of their illness. All were intubated with a 2.5-3.5 mm Coles pattern PVC orotracheal tube (Portex), and radiographic examination showed that the tube tip lay in the mid-trachea.

Whenever a chest radiograph was required for clinical management, the patient was placed in a supine posture and allocated to one of five groups that defined the type of suction catheter to be assessed and its manner of insertion. These were:

Group 1 straight catheter, head straight;

Group 11 straight catheter, head to left;

Group 111 straight catheter, head to right;

Group 1V curved catheter, (tip directed to right), head to left;

Group V curved catheter, (tip directed to left), head to right.

Ten assessments were made in each group.

Catheters used were made of siliconised PVC (Portex) and the size (6 or 8 FG) was determined by the size of the endotracheal tube. When a curved catheter was used a straight line was drawn along the length of the tubing, to ensure that the curved tip remained on the desired side during insertion.

The catheter was introduced by one of us (M P) via the endotracheal tube, with the head in the predetermined position and was gently advanced until resistance was felt. It was then withdrawn minimally, the head returned to the midline, and the radiograph taken. The catheter was removed immediately afterwards. A record was made of the precise procedure carried out, before the radiological findings were known. On the radiograph the suction catheter could be clearly seen entering one or other main bronchus-its location was noted.

\section{Results}

Straight catheters inserted with the patient's head facing forward, more commonly entered the right
Table Location of catheter in relation to head position and type of catheter ( $n=10$ in each group)

\begin{tabular}{llcl}
\hline Type of catheter & Head position & \multicolumn{2}{c}{ Catheter position by radiograph } \\
\cline { 3 - 4 } & & $R M B$ & LMB \\
\hline Straight & Straight & 7 & 3 \\
Straight & Right & 3 & 7 \\
Straight & Left & 10 & 0 \\
Curved tip & Right & 1 & 9 \\
Curved tip & Left & 10 & 0 \\
\hline
\end{tabular}

RMB =-right main bronchus; LMB = left main bronchus.

main bronchus than the left (Table). Turning the head improved the consistency with which a catheter could be directed into the contralateral bronchus, although the left main bronchus was less successfully catheterised than the right. By using a marked, curved tip catheter together with head turning, the catheter failed to enter the contralateral bronchus on only 1 of 20 occasions.

\section{Discussion}

As has been shown in adults, the left main bronchus is more difficult to catheterise than the right because of the more acute angle at which the left mainstem bronchus emerges from the trachea. Our results show that with the head turned to the left both straight and curved catheters can be directed into the right main bronchus. With the head turned to the right, a straight catheter will enter the left side on some occasions, but if a curved tip catheter is used with the tip directed to the left side this will occur more consistently.

In a review of 20 sets of chest radiographs carried out on newborn babies with no obvious lung disease we found that in 19 the left main bronchus subtended a more acute angle with the trachea than did the right. Moreover, this difference between the bronchi was present in five infants who had unilateral atelectasis and persisted after the lung had reinflated. We would therefore expect the accuracy of suction catheter placement to be as successful in patients with unilateral atelectasis as it was in our study group.

It might be expected that a more rigid type of suction catheter would be easier to place with accuracy. An alternative stiffer catheter entered the left main bronchus much less frequently, however, than did the floppier Portex catheter.

All infants in this study were intubated by the orotracheal route. Studies in adults have shown that catheterisation of the left main bronchus is more successful through a tracheostomy. ${ }^{6}$ It is possible that selective catheterisation via a nasotracheal tube might be less successful than via an orotracheal tube. 
We suggest that by means of head positioning a flexible, curved tip suction catheter may be placed reliably in the right or left main bronchus of intubated newborns. By this means, selective bronchial suctioning or lavage can be carried out easily without the need for bronchoscopy or radiographic screening facilities.

\section{References}

1 Whitfield NM, Douglas Jones M. Atelectasis associated with mechanical ventilation for hyaline membrane disease. Crit Care Med 1980;8:729-32.

2 Wyman ML, Kuhns LR. Lobar opacification of the lung after tracheal extubation in neonates. J Pediatr 1977;91: 109-12.
3 Brandstater B, Muallem M. Atelectasis following tracheal suction in infants. Anesthesiology 1969;31: 468-72.

4 Bush GH. Tracheobronchial suction in infants and children. Br J Anaesth 1963;35:322-6.

5 Gregory GA. Respiratory care of newborn infants. Symposium on recent clinical advances. Pediatr Clin North Am 1971;19:311-24.

6 Anthony JS, Sieniewicz DJ. Suctioning of the left bronchial tree in critically ill patients. Crit Care Med 1977;5:161-2.

Correspondence to $\mathrm{Dr} M$ Silverman, Department of Paediatrics and Neonatal Medicine, Hammersmith Hospital, Ducane Road, London W12 0HS.

Received 27 June 1983

\section{Hypoxanthine guanine phosphoribosyl transferase deficiency presenting with gout and renal failure in infancy}

\section{P C HOLLAND, M J DILlON, J PINCOTT, H A SIMMONDS, AND T M BARRATT}

Renal Unit, The Hospital for Sick Children, Great Ormond Street, and Purine Laboratory, Guy's Hospital, London

SUMMARY A month old infant had gout and renal failure caused by hypoxanthine guanine phosphoribosyl transferase (HGPRTase) deficiency. Investigations showed a high uric acid value, crystal nephropathy on ultrasound, and uric acid deposition on renal biopsy. The HGPRTase value was low in red cells and fibroblasts.

The Lesch-Nyhan syndrome ${ }^{1}$ caused by hypoxanthine guanine phosphoribosyl transferase (HGPRTase) deficiency $^{2}$ usually presents in late infancy or early childhood with self mutilating behaviour and retarded development, but in young adults with the milder form of the disease urolithiasis and renal impairment have been reported. ${ }^{3}$ Although this clinical picture is rare in infancy, we describe a child aged five weeks who presented with gout and renal failure, and emphasise the problems encountered in diagnosis and treatment.

\section{Case report}

A baby boy, the first child of healthy unrelated parents, was born at 39 weeks' gestation, after a normal pregnancy, and weighed $3 \cdot 2 \mathrm{~kg}$. From age three weeks he fed poorly and was excessively irritable. On admission to hospital, irritability was confirmed and the interphalangeal joints of the first, second, and third fingers of the left hand were red, swollen, and obviously painful (Fig. 1). Abdominal examination was normal, blood pressure was $95 / 65$ $\mathrm{mmHg}$, and examination of the central nervous system showed mild hypotonia with normal neonatal reflexes. A maternal grandfather had suffered

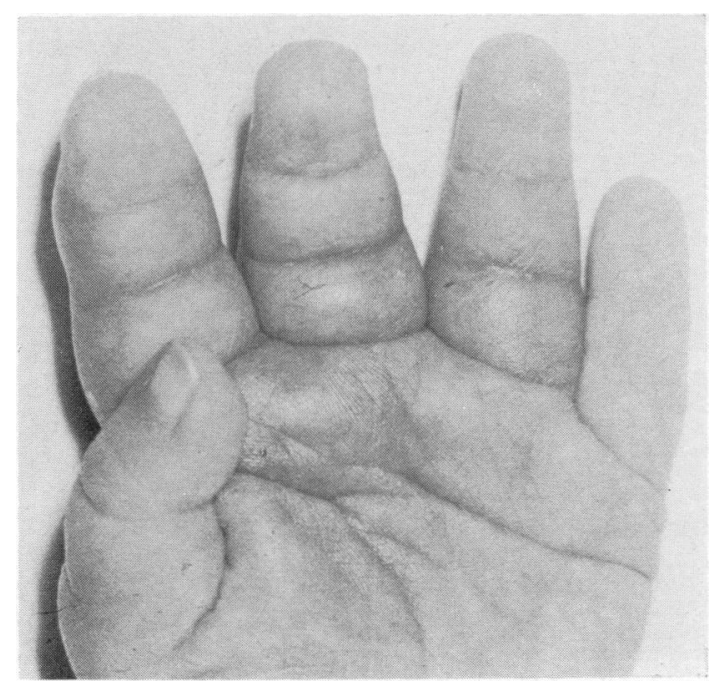

Fig 1 Swollen interphalangeal joints of the 1st, 2nd, and 3rd fingers of the left hand. 